\title{
A Case of Gingival Metastasis from Rectal Cancer in Which Immunohistochemistry and PET-CT Were Useful for the Diagnostic Procedure
}

\author{
Masami Yamauchi ${ }^{a}$ Katsunori Shinozaki ${ }^{a}$ Mihoko Doi ${ }^{a} \quad$ Tomoko Nitta $^{a}$ \\ Takashi Nishisaka ${ }^{\text {b }}$ \\ Divisions of a Clinical Oncology and ${ }^{b}$ Pathology, Hiroshima Prefectural Hospital, \\ Hiroshima, Japan
}

\section{Key Words}

Gingival metastasis $\cdot$ Colorectal cancer $\cdot$ Immunohistochemistry $\cdot$ PET-CT

\begin{abstract}
A 50-year-old man was referred to our hospital because of a 2-month history of painful gingival swelling. Histopathological examination of the biopsy specimen showed a metastatic adenocarcinoma, and a chest-abdominopelvic CT showed multiple metastases in the lung, liver, and spleen, but failed to demonstrate the primary tumor. He had never complained of abdominal symptoms, and physical examination did not show any abnormality in the abdomen. However, immunohistochemical staining including caudal-related homeobox transcription factor (CDX-2) of the gingival tumor and PET-CT findings strongly suggested colorectal cancer as the origin. Colonoscopy then revealed a tumor in the rectum, and systemic chemotherapy was started immediately.

(c) 2014 S. Karger AG, Basel
\end{abstract}

\section{Introduction}

Cancer metastases to the oral cavity are extremely rare, with a reported prevalence of only $1-2 \%$ of all oral malignant tumors. Meanwhile, recent reports have shown that the primary cancers of oral and oropharyngeal metastatic tumors can be found in various organs [1]. Therefore, the workup for detecting the primary is confusing, especially among cases in

Masami Yamauchi

Division of Clinical Oncology

Hiroshima Prefectural Hospital

1-5-54 Ujinakanda, Minami-ku, Hiroshima 734-8530 (Japan)

E-Mailmt.28@nifty.com 
Yamauchi et al: A Case of Gingival Metastasis from Rectal Cancer in Which Immunohistochemistry and PET-CT Were Useful for the Diagnostic Procedure

which patients remain asymptomatic at the time of the initial diagnosis. The present case report shows the usefulness of combined interpretation of immunohistochemistry and PETCT for the prompt diagnosis of the primary lesion in this unusual clinical setting.

\section{Case Report}

A previously healthy 50-year-old man presented with an approximately 2-month-long history of lower jaw pain. He had no history of smoking and alcohol overconsumption. He first visited the dental clinic, and the dentist, suspecting gingival cancer, referred the patient to our hospital.

On clinical examination, an ulcerative, circumferential tumor that arose in the left lower gingiva was visible (fig. 1). Physical examination of the chest and abdomen was normal. The laboratory findings were as follows: white blood cell count $6,800 / \mathrm{mm}^{3}$; hemoglobin 13.9 $\mathrm{g} / \mathrm{dl}$; LDH $326 \mathrm{IU} / \mathrm{l}$, and increased CEA and CA19-9 levels $(215 \mathrm{ng} / \mathrm{ml}$ and $339 \mathrm{U} / \mathrm{ml}$, respectively). Other findings were within the normal limits.

Because biopsies obtained from the gingival tumor confirmed adenocarcinoma with a substantial gland formation, CT was performed to determine the primary and the extent of the disease. It revealed small nodular masses in the lung, liver, and spleen, all of which were round in shape, $2-3 \mathrm{~cm}$ in diameter, with clear borderlines, suggesting metastases. However, CT gave no clues about the primary tumor.

On the fifth day after the referral, the results of the immunohistochemical staining for adenocarcinoma turned out to be positive for CK20 and caudal-related homeobox transcription factor (CDX-2), and negative for CK7 and thyroid transcription factor-1 (TTF-1) (fig. 2). Moreover, PET-CT showed an accumulation of ${ }^{18}$ F-fluorodeoxyglucose (FDG) with an SUV max of 19.0 in the upper rectum (fig. 3a). Based on these additional findings, the patient was persuaded to undergo a colonoscopic examination, which then revealed advanced rectal cancer (fig. 3b). After the patient received 16 cycles of modified FOLFOX6 (fluorouracil 400 $\mathrm{mg} / \mathrm{m}^{2}$ intravenous bolus on day 1 , followed by continuous infusional fluorouracil 2,400 $\mathrm{mg} / \mathrm{m}^{2}$ administered over $46 \mathrm{~h}$ starting on day 1 in combination with leucovorin $200 \mathrm{mg} / \mathrm{m}^{2}$ on day 1 and oxaliplatin $85 \mathrm{mg} / \mathrm{m}^{2}$ on day 1) plus bevacizumab treatment, the rectal cancer and the gingival metastasis disappeared, and all other metastases shrank. Since then, 12 months have passed, and he is now undergoing second-line chemotherapy with FOLFIRI (fluorouracil $400 \mathrm{mg} / \mathrm{m}^{2}$ intravenous bolus on day 1 , followed by continuous infusional fluorouracil $2,400 \mathrm{mg} / \mathrm{m}^{2}$ administered over $46 \mathrm{~h}$ starting on day 1 in combination with leucovorin $200 \mathrm{mg} / \mathrm{m}^{2}$ on day 1 and irinotecan $150 \mathrm{mg} / \mathrm{m}^{2}$ on day 1) plus bevacizumab.

\section{Discussion}

The oral region is an uncommon site for malignant tumor metastasis. The most common primary sites are the lung, breast, and kidney. These 3 neoplasms account for approximately $40 \%$ of all oral metastases. The remaining cases consist of miscellaneous primary malignancies (i.e. bone, large bowel, skin, adrenal gland, liver, uterus, ovaries, and stomach) [2]. Because of the rarity and variety of its presentation, the differential diagnosis and recognizing the primary tumor are challenging to clinicians.

In $24 \%$ of patients, the metastatic lesion in the oral region is the first indication of an undiscovered malignancy at a distant site [2]. If careful history taking, physical examination, and standard laboratory tests do not show conclusive evidence of a primary tumor, the 
diagnostic strategy for unknown primary tumors could be helpful [3]. In this strategy, it is generally accepted that invasive procedures such as bronchoscopy, upper endoscopy, and colonoscopy cannot be recommended for routine workups. A CT should first be considered because it is widely available and well established in diagnosing and staging cancers, but its limited sensitivity at the initial workup stage has been pointed out [4]. Therefore, the NCCN Guidelines for the occult primary emphasize the importance of the detailed immunohistochemical interpretation of the biopsy samples and its use in conjunction with appropriate imaging studies [5].

Immunohistochemical staining is useful for the characterization of tumor cells and the efficient pathologic diagnosis. However, immunohistochemical markers are not uniformly specific or sensitive, and a large series of marker studies should be avoided. In the present case, markers were chosen based on the light microscopic findings that suggested the possibility of gastrointestinal neoplasm. Low-molecular-weight cytokeratins, CK7 and CK20, are the most common stains to define subsets of carcinomas, and CK20+/CK7- favors colorectal primary tumors. TTF-1 excludes the possibility of lung tumors, and CDX-2 strengthens the diagnosis of colorectal cancer because it is regarded as being highly specific for the intestinal epithelium [6].

Compared to immunohistochemistry, the role of PET-CT for detecting an undiscovered primary lesion is controversial. PET-CT is noninvasive and certainly detects more primary sites than conventional imaging techniques $[7,8]$. However, its exact role remains undefined because of the lack of prospective clinical trials comparing PET-CT with conventional imaging modalities. Clinicians should also be aware that FDG uptake is not tumor-specific and can be observed in benign tissues with inflammation or trauma. As shown in the present case, nodular high FDG uptake in the colon and rectum is predictive of malignant pathologic findings [9]. Taking the results of immunohistochemical staining into account, colonoscopy was a reasonable next step for further diagnostic evaluation of the present patient, even though he never had had gastrointestinal symptoms.

In conclusion, the combined use of immunohistochemical examinations and PET-CT findings may play a pivotal role for diagnostic workup to detect the primary cancer in a rare case with gingival metastasis. Prompt and accurate diagnosis leads to appropriate therapeutic management with effective modern chemotherapy.

\section{Disclosure Statement}

The authors declare no conflict of interest.

\section{References}

1 Shin SJ, Roh JL, Choi SH, Nam SY, Kim SY, Kim SB, Lee SW, Cho KJ: Metastatic carcinomas to the oral cavity and oropharynx. Korean J Pathol 2012;46:266-271.

2 Hirshberg A, Shnaiderman-Shapiro A, Kaplan I, Berger R: Metastatic tumours to the oral cavity pathogenesis and analysis of 673 cases. Oral Oncol 2008;44:743-752.

-3 Varadhachary GR, Abbruzzese JL, Lenzi R: Diagnostic strategies for unknown primary cancer. Cancer 2004;100:1776-1785.

4 Abbruzzese JL, Abbruzzese MC, Lenzi R, Hess KR, Raber MN: Analysis of a diagnostic strategy for patients with suspected tumors of unknown origin. J Clin Oncol 1995;13:2094-2103.

-5 Ettinger DS, Agulnik M, Cates JM, Cristea M, Denlinger CS, Eaton KD, Fidias PM, Gierada D, Gockerman JP, Handorf CR, Iyer R, Lenzi R, Phay J, Rashid A, Saltz L, Shulman LN, Smerage JB, Varadhachary GR, Zager JS, Zhen WK: National Comprehensive Cancer Network: occult primary. J Natl Compr Canc Netw 2011;9:13581395. 


\section{Case Reports in Oncology}

\begin{tabular}{l|l}
\hline Case Rep Oncol 2014;7:246-251 \\
\hline DOI: $10.1159 / 000362185$ & $\begin{array}{l}\text { C 2014 S. Karger AG, Basel } \\
\text { www.karger.com/cro }\end{array}$ \\
\hline
\end{tabular}

Yamauchi et al.: A Case of Gingival Metastasis from Rectal Cancer in Which Immunohistochemistry and PET-CT Were Useful for the Diagnostic Procedure

66 Werling RW, Yaziji H, Bacchi CE, Gown AM: CDX2, a highly sensitive and specific marker of adenocarcinomas of intestinal origin: an immunohistochemical survey of 476 primary and metastatic carcinomas. Am J Surg Pathol 2003;27:303-310.

7 Delgado-Bolton RC, Fernández-Pérez C, González-Maté A, Carreras JL: Meta-analysis of the performance of 18F-FDG PET in primary tumor detection in unknown primary tumors. J Nucl Med 2003;44:1301-1314.

$\$ 8$ Hemminki K, Liu H, Heminki A, Sundquist J: Power and limits of modern cancer diagnostics: cancer of unknown primary. Ann Oncol 2012;23:760-764.

-9 Tatlidil R, Jadvar H, Bading JR, Conti PS: Incidental colonic fluorodeoxyglucose uptake: correlation with colonoscopic and histopathologic findings. Radiology 2002;224:783-787.

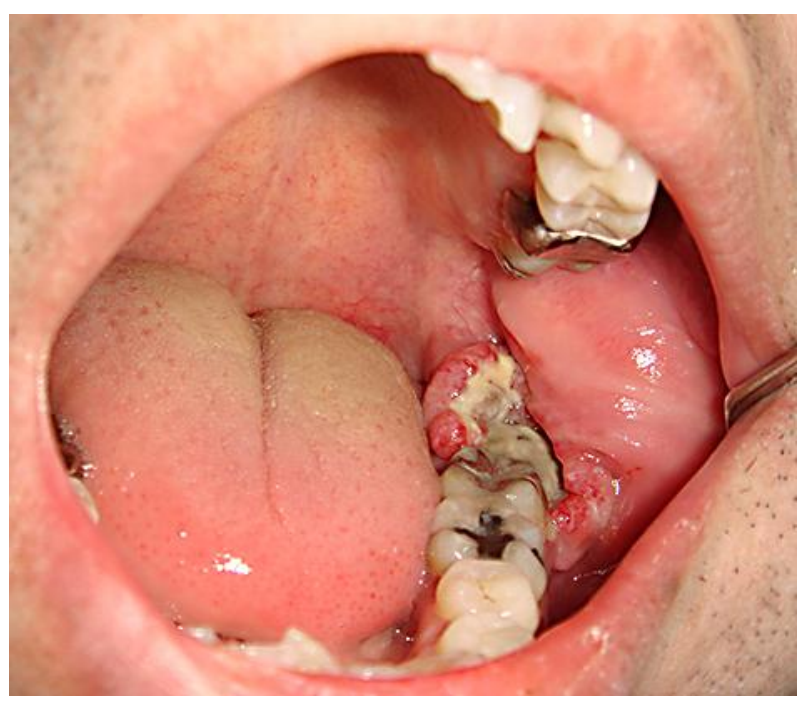

Fig. 1. Inspection reveals an ulcerated tumor in the left lower gingiva. 

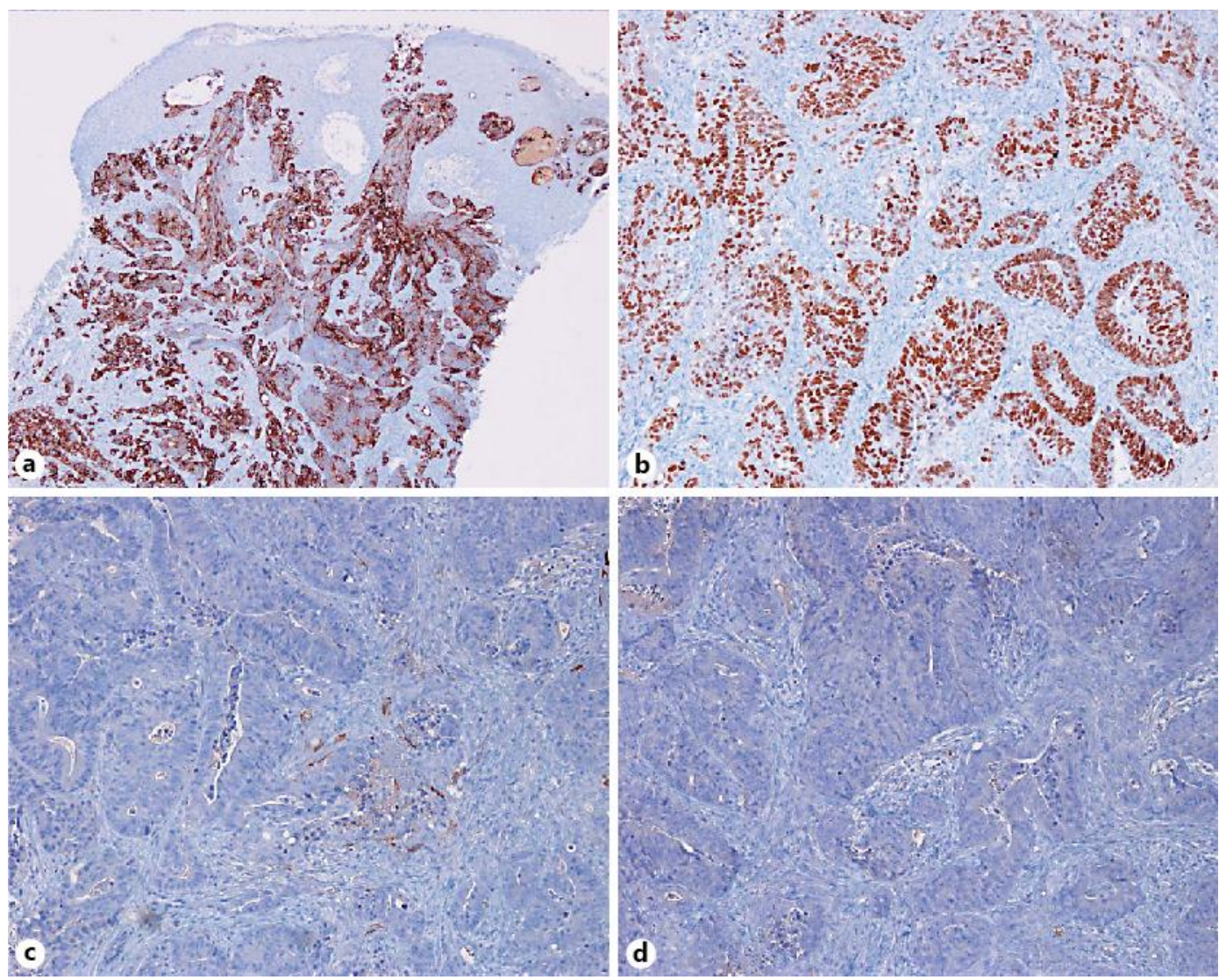

Fig. 2. Immunohistochemical examinations for adenocarcinoma are positive for CK20 (a) and CDX-2 (b), but negative for CK7 (c) and TTF-1 (d). 


\section{Case Reports in Oncology}

\begin{tabular}{l|l}
\hline Case Rep Oncol 2014;7:246-251 \\
\hline DOI: $10.1159 / 000362185$ & $\begin{array}{l}\text { C 2014 S. Karger AG, Basel } \\
\text { www.karger.com/cro }\end{array}$ \\
\hline
\end{tabular}

Yamauchi et al.: A Case of Gingival Metastasis from Rectal Cancer in Which Immunohistochemistry and PET-CT Were Useful for the Diagnostic Procedure
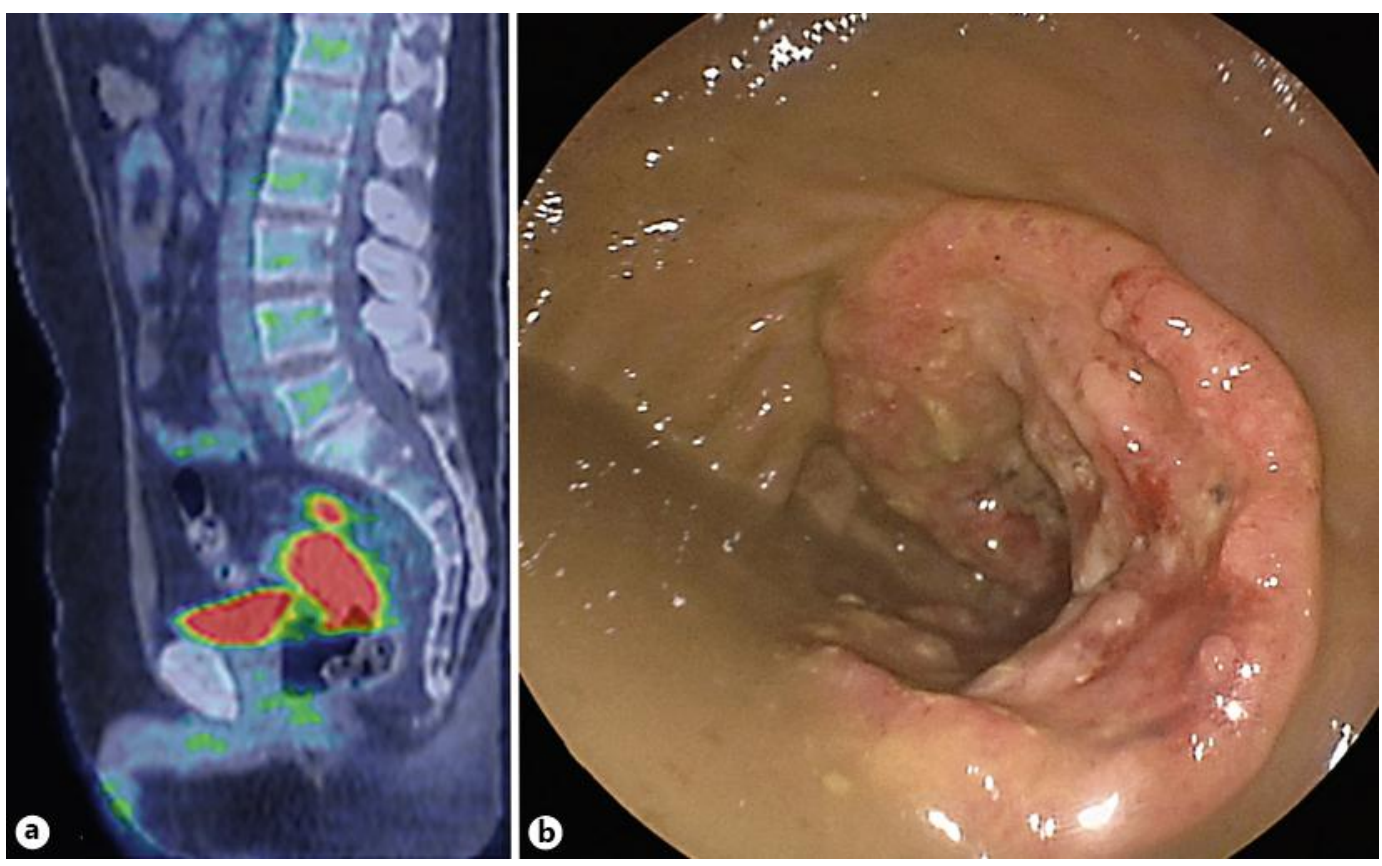

Fig. 3. a PET/CT with sagittal reconstruction reveals a large amount of FDG uptake behind the bladder. $\mathbf{b}$ Colonoscopy demonstrates a circular ulcerative tumor in the upper rectum. 